\title{
émulations
}

\section{Quentin Deluermoz, Pierre Singaravélou - Pour une histoire des possibles. Analyses contrefactuelles et futurs non advenus}

\section{Matthias Fringant}

Émulations - Revue de sciences sociales

2016, «Comptes rendus critiques, En ligne »

\section{Article disponible à l'adresse suivante}

https://ojs.uclouvain.be/index.php/emulations/article/view/7243

\section{Pour citer cet article}

Matthias Fringant, «Quentin Deluermoz, Pierre Singaravélou — Pour une histoire des possibles. Analyses contrefactuelles et futurs non advenus », Émulations, en ligne. Mise en ligne le 3 octobre 2016.

DOI : 10.14428/emulations.cr.026

Distribution électronique : Université catholique de Louvain (Belgique) : ojs.uclouvain.be

(c) Cet article est mis à disposition selon les termes de la Licence Creative Commons Attribution, Pas d'Utilisation Commerciale 4.0 International. http://creativecommons.org/licenses/by-nc/4.0/

Éditeur : Émulations - Revue de sciences sociales / Presses universitaires de Louvain https://ojs.uclouvain.be/index.php/emulations

ISSN électronique : $1784-5734$

UCL PRESSES 


\section{Quentin Deluermoz, Pierre Singaravélou - Pour une histoire des possibles. Ana- lyses contrefactuelles et futurs non adve-}

Matthias Fringant ${ }^{1}$

Recensé: Quentin Deluermoz, Pierre Singaravélou, Pour une histoire des possibles. Analyses contrefactuelles et futurs non advenus, Paris, Seuil ("L'univers historique »), 2016, 448 p.

La question des possibles et, plus largement, celle de l'imaginaire semblent être rétives aux sciences sociales. Elle a ainsi pu susciter de la méfiance, parfois renforcée par les dérives de son traitement dans ses formes les plus "postmodernes» (Lahire, 2007; Quinon, Saint-Martin, 2016). C'est pourtant ce difficile objet que les historiens Quentin Deluermoz et Pierre Singaravélou font ici le pari de disséquer, en étudiant de quelle manière il est envisageable de recourir au raisonnement contrefactuel — «Que se serait-il passé si... ?»— en histoire et, plus largement, en sciences sociales. Loin des écueils précédemment mentionnés, ils réalisent avec brio la démonstration selon laquelle un usage savant et rigoureux du contrefactuel est possible et, sous certaines conditions, souhaitable, ouvrant du même coup un domaine d'études relativement marginal en France $^{2}$. Restituant aux lecteurs les résultats de six années de travail collectif suivant l'ordre chronologique de leur enquête, les deux chercheurs abordent ainsi la notion de contrefactuel en trois étapes.

Le premier temps de l'enquête dresse ainsi un tableau extrêmement dense des usages du raisonnement contrefactuel, à travers quatre grands ensembles : la pratique ancienne dans le travail d'historien (chapitre 1); la diffusion internationale de ce type d'histoire (chapitre 2); sa variante littéraire et uchronique (chapitre 3); les usages de ce raisonnement en sciences sociales et physiques (chapitre 4).

La démarche contrefactuelle, apparue dès le $\mathrm{V}^{\mathrm{e}}$ siècle av. J.-C. sous la forme de la digression, ne deviendra une véritable pratique d'écriture qu'au début du XIXe siècle sous

\footnotetext{
${ }^{1}$ Doctorant en sociologie au Centre européen de sociologie et de science politique (CESSP), École des hautes études en sciences sociales de Paris (EHESS).

2 Signalons toutefois la parution en janvier 2015 d'un ouvrage français traitant également de l'histoire contrefactuelle, d'ailleurs préfacé par Quentin Deluermoz et Pierre Singaravélou (Besson, Synowiecki, 2015).
} 
la forme de l'uchronie, synonyme d'utopie dans l'histoire. En ce qui concerne plus précisément les sciences sociales, c'est à Max Weber qu'il revient d'avoir théorisé en premier la notion, dans ses Essais sur la théorie de la science publiés en 1906. Relativement marginale au cours du XXe siècle malgré certains développements notables tels que la cliométrie américaine représentée par Robert Fogel au cours des années 1950, la tentative fondatrice d'institutionnalisation d'une démarche résolument scientifique sera effectuée par l'historien britannique Niall Ferguson et la publication de Virtual History en 1997, menant à une normalisation de son usage au cours des années 2000.

Si le moteur de la version qui se dit la plus scientifique de l'histoire contrefactuelle semble donc être le monde anglo-saxon, une circulation internationale de ce type d'histoire s'est accomplie au cours du $\mathrm{XX}^{\mathrm{e}}$ siècle, notamment dans des régions sous influences britannique ou états-unienne, comme l'Inde, l'Australie, l'Océanie, ou encore l'Amérique latine. L'histoire contrefactuelle s'est également exportée en Europe; la France faisant exception en raison d'aspects scientifique, académique, et social, expliquant ainsi la marginalité de ce domaine d'études, que les auteurs contribuent par le présent ouvrage à combler.

L'autre pendant de l'histoire contrefactuelle est sa version littéraire, ou uchronique. Quentin Deluermoz et Pierre Singaravélou proposent ici une synthèse de son développement, en rappelant les liens flous de réciprocité entre raisonnements contrefactuels historien et littéraire contribuant à expliquer la méfiance des historiens à l'égard de la notion. Notons ici que si les auteurs font le choix d'élargir l'analyse aux formes populaires du contrefactuel, ils évitent sans difficulté l'écueil de ne voir dans celles-ci qu'une variante appauvrie du pan le plus savant de la même notion, sans toutefois occulter les rapports existant entre les deux (Grignon, Passeron, 1989).

Enfin, en mettant ce type de raisonnement à l'épreuve des sciences sociales et physiques, les auteurs concluent ce premier tour d'horizon, en affirmant que les usages du raisonnement contrefactuel « appartiennent à des domaines disparates d'expression, du savoir ou de la vie courante. Cette diversité suscite bien des confusions, des incertitudes, des rejets et des adhésions enthousiastes » (p. 98). Dès lors, la question ne semble plus être de savoir s'il faut ou non mobiliser ce raisonnement en histoire, puisqu'il semble inévitable, mais plutôt en expliciter les questions sous-jacentes.

La deuxième partie s'attache donc logiquement à examiner de manière détaillée ces problématiques, mais aussi à «préciser les conditions auxquelles elle peut s'avérer utile» (p. 101) pour l'enquête historique. Quatre domaines d'interrogation sont ici envisagés : le rôle de l'imagination (chapitre 5); la question de la causalité (chapitre 6); les formes d'historicité (chapitre 7); les enjeux politiques et sociaux du travail d'historien (chapitre 8).

En ce qui concerne les liens entre imagination et pratique du travail d'historien, un rapide rappel permet aux auteurs d'affirmer que «la question qui agite les débats n'est donc pas tant de choisir entre science et littérature que de savoir où placer le curseur 
entre ces deux pôles» (p. 105). Ainsi, il apparaît que «l'imagination, une fois sous contrainte, appartient pleinement aux modalités de l'enquête historique» (p. 114), permettant aussi bien d'aider à la contextualisation que de cerner les manières de penser des sociétés étudiées ou encore d'imaginer plusieurs futurs non advenus permettant de restituer la complexité du passé. Enfin, les deux historiens indiquent que cette démarche entretient des liens étroits avec la réflexivité, en ce qu'il semble possible «d’y recourir pour expliciter, et de ce fait mettre à distance, son propre investissement dans la pratique de recherche, habituellement masqué dans l'écriture finale» (p. 124). Quentin Deluermoz et Pierre Singaravélou se situent ici nettement du côté de la réflexivité conçue comme « objectivation du sujet de l'objectivation »(Bourdieu, 2003), laissant de côté les usages plus problématiques du même terme (Gingras, 2004).

Un tour d'horizon historique et épistémologique du problème de la causalité en sciences sociales mène les auteurs à rappeler que la question principale en la matière est que les chercheurs analysent un fait qui a toujours une multiplicité de causes. Une fois cette idée acquise, des tensions résistent, telles que la question du continuum entre déterminisme et contingence ou celle de la continuité et de la rupture. Mobilisant Max Weber, les auteurs listent la multiplicité des réflexions sur la causalité mobilisant le contrefactuel, l’idée principale étant celle selon laquelle «l'approche contrefactuelle est par définition une pensée des relations; en tant que principe, elle est indéterminée et permet de réfléchir à nouveaux frais sur ces questions » (p. 151).

La question de l'historicité vise, quant à elle, à savoir si le raisonnement contrefactuel peut s'appliquer de manière universelle (à toute époque et à toute société), ou bien s’il est au contraire dépendant des caractéristiques de l'Occident moderne. En mobilisant différents travaux classiques d'anthropologie sur les rapports au temps de différentes sociétés « lointaines », l'argument principal est que le raisonnement contrefactuel sous les formes que nous connaissons est assujetti aux régimes d'historicité de l'Occident moderne. Cependant, en soulignant la plasticité de la notion, les auteurs évoquent des alternatives permettant de lui faire jouer le rôle de révélateur de divers rapports au temps. Enfin, la question des enjeux politiques du travail d'historien revient à se demander si l'histoire contrefactuelle serait ou non le lieu privilégié des idéologies assumées ou impensées de leurs auteurs. La réponse ici proposée consiste à dire que, loin de se réduire à une idéologie unique, l'emploi de cette démarche a, au cours du temps, recoupé des usages politiques divers dans des contextes toujours variés.

Refusant - en acte - une séparation tranchée entre théorie et pratique souvent dénoncée comme néfaste à la constitution du savoir scientifique (Bourdieu, 1980), Quentin Deluermoz et Pierre Singaravélou proposent dans un dernier temps de restituer aux lecteurs trois expérimentations leur permettant d'évaluer concrètement la pertinence du contrefactuel : une première sur l'histoire globale adoptant une perspective macro permettant de mettre au jour la capacité de ce raisonnement à « déconstruire les schémas historiographiques classiques » (p. 247) (chapitre 9); une seconde sur la révolution parisienne de février-juin 1848 adoptant une perspective plus micro et réaffirmant la 
capacité de cette démarche à " aborder et de restituer autrement un phénomène qui semble encore mettre à mal bien des implicites du récit de l'historien » (p. 282) (chapitre 10); et, enfin, une dernière centrée autour la question des modes de transmission et de partage des savoirs promouvant les dimensions pédagogiques de ce type de raisonnement, faisant appel à la participation, à l'échange et aux affects (chapitres 11 et 12).

Au terme de cette enquête se dégage une esquisse de cadre conceptuel à la fois simple, mais relativement efficace pour l'usage du raisonnement contrefactuel dans la pratique historienne, mais aussi, plus largement, dans l'ensemble des sciences sociales. L'histoire est, à de multiples reprises, considérée comme faisant partie du champ unifié des sciences historiques (Passeron, 1991) à l'heure où l'hyperspécialisation tend à nuire à la construction du savoir sur l'homme (Lahire, 2012). Les auteurs spécifient les usages qui ont paru les plus pertinents de ce raisonnement, au nombre de six : le contrefactuel réflexif, qui permet de formuler clairement les contrefactuels implicites que comportent sans le dire des raisonnements ; le contrefactuel de contextualisation, qui permet de comprendre ce qui a été en interrogeant ce qui n’a pas été; le contrefactuel des causalités et des interdépendances, qui permet de penser le possible et l'impossible des trajectoires; le contrefactuel de mise en intrigue, qui sert à débusquer dans les trames narratives les schèmes implicites du discours historique; le contrefactuel des futurs non advenus, explorant la façon dont les acteurs se représentaient leur propre avenir ou les futurs de leur passé; le contrefactuel des possibles du passé, permettant d'appréhender les virtualités réelles des situations révolues.

Ces différents usages peuvent s'exercer dans le cadre de trois temporalités : longue, moyenne, et courte. La dernière semble, selon les auteurs, être la plus utile pour les chercheurs en sciences sociales. En dernière analyse, «ces trois temporalités et six usages constituent un cadre d'analyse qui permet de distinguer les différents contrefactuels en fonction de l'objectif visé et d'éviter les confusions qui ont suscité à juste titre la réprobation des historiens» (p. 345).

Cet ouvrage constitue en définitive une prise de position forte et réellement convaincante pour le développement d'une histoire contrefactuelle rigoureuse, permettant de stimuler «l'imagination historique » à nouveaux frais (Wright Mills, 2006)3. Ce travail collectif et de longue haleine a le mérite d'offrir une argumentation à la fois érudite, claire malgré l'énumération parfois un peu longue de références pouvant nuire à la compréhension du raisonnement global, et savamment construite. Elle résulte par ailleurs d'une enquête empirique importante, dont les conditions de production sont clairement explicitées. Cette exigence globale évite donc les écueils les plus fréquents auxquels peuvent être associés certains travaux contemporains en sciences sociales. Sans

3 C. Wright Mills proposait, dans L'imagination sociologique, de substituer, en fonction des besoins, le terme «sociologique» par différentes sciences sociales, ce que nous nous permettons donc de faire ici. 
prétendre à un quelconque « tournant contrefactuel » qui viendrait s'ajouter à la longue liste de ceux s'étant succédé depuis les années 1970, les auteurs permettent de mettre à disposition des praticiens des sciences historiques un nouvel outil qui, nécessitant d'être utilisé sous certaines conditions ici esquissées, peut s'ajouter et se combiner à d'autres. En effet, les auteurs ayant souligné les liens que le contrefactuel entretient avec la réflexivité, il peut être bon de rappeler la pertinence de cette dernière, récemment défendue dans une conception similaire par d'autres chercheurs (Weber, 2015).

En définitive, si la science a pour enjeu le monopole de la représentation scientifiquement légitime du réel (Bourdieu, 2001), il semble que l'inclusion de l'imaginaire et des possibles passés dans cette lutte ait ici reçu un coup d'envoi magistral qu'il sera difficile pour les sciences sociales d'ignorer à l'avenir.

\section{Bibliographie}

BESSON F., SyNOWIECKI J. (2005), Écrire l’histoire avec des «si », Paris, Éditions Rue d’Ulm («Actes de la recherche à l’ENS »).

BOURDIEU P. (1980), Le sens pratique, Paris, Éditions de Minuit («Le sens commun »).

Id. (2001), Science de la science et réflexivité, Paris, Raisons d’agir ("Cours et travaux»).

Id. (2003) [1997], Méditations pascaliennes, Paris, Seuil («Points essais»).

GRIGNON C., PASSERON J.-C. (1989), Le savant et le populaire. Misérabilisme et populisme en sociologie et en littérature, Paris, Gallimard/Le Seuil (« Hautes Études»).

GingRAS Y. (2004), « Réflexivité et sociologie de la connaissance scientifique », in L. Pinto, G. Sapiro, P. Champagne, M.-C. Riviere (dir.) Pierre Bourdieu, sociologue, Paris, Fayard (« Histoire de la pensée »), p. 337-347.

LAHIRE B. (2007 [2005]), «Une astrologue sur la planète des sociologues ou comment devenir docteur en sociologie sans posséder le métier de sociologue » ? in L'esprit sociologique, Paris, La Découverte («La Découverte/Poche »), p. 351-387.

Id. (2012), Monde pluriel. Penser l’unité des sciences sociales, Paris, La Découverte («La couleur des idées »).

PASSERON J.-C. (2006 [1991]), Le raisonnement sociologique. Un espace non poppérien de l'argumentation, Paris, Albin Michel («Bibliothèque de “L’Évolution du l'humanité"»).

QUiNON M., SAINT-MARTIN A. (2016), Le sociologue imaginaire. Tribulations du professeur Michel Maffesoli et de ses créatures, Marseille, Agone («Mémoires sociales »). 
Weber F. (2015), Brève histoire de l'anthropologie, Paris, Flammarion ("Champs essais »).

WRIGHT Mills C. (2006 [1967]), L'imagination sociologique, Paris, La Découverte («Poche/Sciences humaines et sociales »). 